\title{
Síndrome de Hanhart
}

\author{
Dra. Silvia Castillo T.' : Dr. Jorge Rojas $Z^{2}{ }^{2}$ : Dr. Luis Monasterio A. ${ }^{2}$
}

\section{Hanhart Syndrome}

The case of a male infant with oral and limb abnormalities consistent with the Hanhart or aglossia-adactylia syodrome is reported. There are other cases of developmental disturbances in his family. The aetiology of this syndrome is unknown and there is an extreme variability in its clinical manifestations. Considerations relaţive to embrionic development which may be sesponsible for the tindings in this syndrome are discussed.

(Key words: Adactylia. Aglossia. Hanhart Syndrome).

- El síndrome de Hanhart o aglosia-adactilia, se caracteriza por ausencia en grado variable de porciones distales de una o más extremidades, junto con micrognasia o microglosia severa o ambas.

El primer caso de ausencia congénita de la lengua fue descrito en 1718 por De Jussieu, en una niña de 15 años con un rudimento de aspecto cicatricial en el lugar de la lengua, que la permitía deglutir y expresarse limitadamente ${ }^{1}$. Kettner en 1907 fue el primero en comunicar la asociación de aglosia y severas anomalías en pies y manos en un niño de 4 años, en el que se habia seccionado al nacer una gruesa banda glosopalati$\mathrm{na}^{2}$. En 1932 Rosentha] describió otro caso de aglosia--adactilia y hasta 1975 se habian reunido alrededor de 20 casos en la literatura mundial ${ }^{1-3-4-5-6}$.

1. Becada de Genética, Facultad de Medicina, Hospital J.J. Aguirre, Uniyersidad de Chile.

2. Cirujanos Plásticos, Unidad de Fisurados Clínica Alemana, Unidad Cìrugía Plástica Hospital de Nínos Roberto del Río.
El sindrome de Hanhart es extraordinariamen. te infrecuente, pero es importante describirlo para que sea posible detectarlo y de esta manera contribuir al esclarecimiento de sus posibles factores etiológicos, hereditarios o ambientales. Debe buscarse los defectos orales al examinar a un paciente con malformaciones distales de las ex. tremidades.

En el diagnóstico diferencial de este sindrome deben considerarse la ectrodactilia y las parálisis aisladas de nervios craneales, también el sindrome de Poland Möbius, que es la combinación completa o incompleta de sinbraquidactilia, defecto torácico y parálisis de un nervio craneal. El síndrome de bandas amnióticas se distingue de la agenesia primaria de extremidades por la presencia de cicatrices activas en el recièn nacido, con anillos que constriñen e interrupción de los dermatoglifos por la amputación ${ }^{7-8}$.

\section{Caso clínico}

Lactante del sexo masculino, nació por cesárea debido a sufrimiento fetal, luego de un 


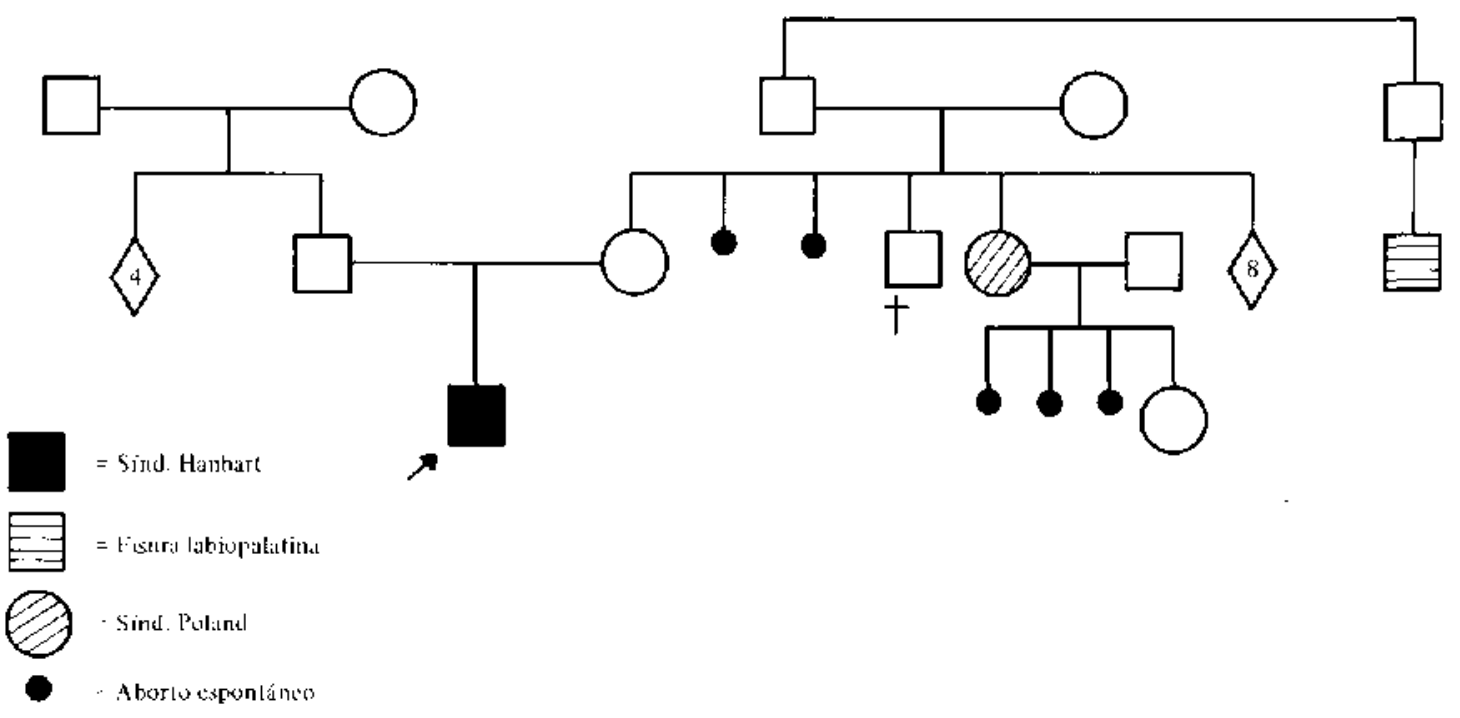

Figura I. Genealogra del paciente com sindrome de ltanthat.

embarazo aparentemente de término sin incidentes. No fue posible descartar la ingestión de thedicamentus durante la gestación. Poco después de nacer fue sometido a una intervención quirúrgica de urgencia para paliar una severa malformación de la boca, con el propósito de lograr la apertura de ésta. En la intervención suffíó un paro cardíaco del que se resuperó. Su genealogía se muestra en la Figura 1 . Su madre de 26 años tenia menstruaciones muy irregulares, es radio operadura de un hospital de provincia, había tenido cuatro cmbarazos previos, tres terminaron en abortos espontíneos en el primer trimestre $y$ uno en parto normal, una hermana viva, sana. La abuela materna tuvo 13 embarazos, 2 abortos espontáneos en el primer trimestre, 11 partos: una hermana materna tiene en el antebrazo derecho menor diámetro que en el izquierdo, con sindactilia de cuatro dedos y agenesia del múscubo pectoral del mismo lado, por lo cual se le diagnosticó Síndrome de Palaud. Un tio segundo por parte đel abuelo materno tiene labio leporino $y$ fisura palatina.

A la edad de $21 / 2$ meses, la paciente pesaba $3.850 \mathrm{~g}$, su talja era de $55 \mathrm{~cm}$, circunferencia de cráneo $37 \mathrm{~cm}$. Fácilmente irritable, había evidencia de hipertrofia nuscular. Bregma pequeño; orejas grandes, de implantación baja, epicanto, estrabismo convergente bilateral, nariz aguileña; microstomia, fisura del velo palatino y del tercio posterior del paladar óseo; microglosia, lengua fija en la base; masas sublinguales, sinequias alveolares. Micrognasia severa; parálisis faccial bilateral. Cuello rígido. Hernia umbilical con anillo de $2,5 \mathrm{~cm}$ de diámetro. Ectrodactilia con ausencia de los tres dedos centrales de ambas manos. Agenesia de metatarso y ortejos de la extrenidad inferior derecha. El pie izquierdo estaba constituido por cl talón y tres mamelones rudimentarios (Figs. 2, 3 y 4). No se encontraron alteraciones en el tórax ni en los genitales. Desarrollo psicomotor retrasado, incoordinación evidente de la deglución.

\section{DISCUSION}

En el síndrome de Hanhart, la gravedad del compromiso varía en imporancia en las diferentes extremidades, desde peromelia completa hasta ausencia distal de un dedo, sindactilia o hipoplasia ungueal. La micrognasia y lá microglosia pueden ser severas y estar asociadas con fisura palatina, sinequias, signasia, oligodoncia y microstomia. Puede haber parálisis congénita de nervios craneanos uni o bilateral. Habitualmente fa inteligencia es normal $8-9-10-11-12 \cdot 13$.

La comprensión del desarrollo embriológico es importante para entender la asociación de ciertos rasgos en este síndrome:

Entre los días 20 y 30 , el mesodermo paraxial a cada lado de la notocorda se segmenta y forma la base para el desarrollo del esqueleto axial y la musculatura. Durante este período, se esbozan los arcos branquiales y comienza el desarrollo de 


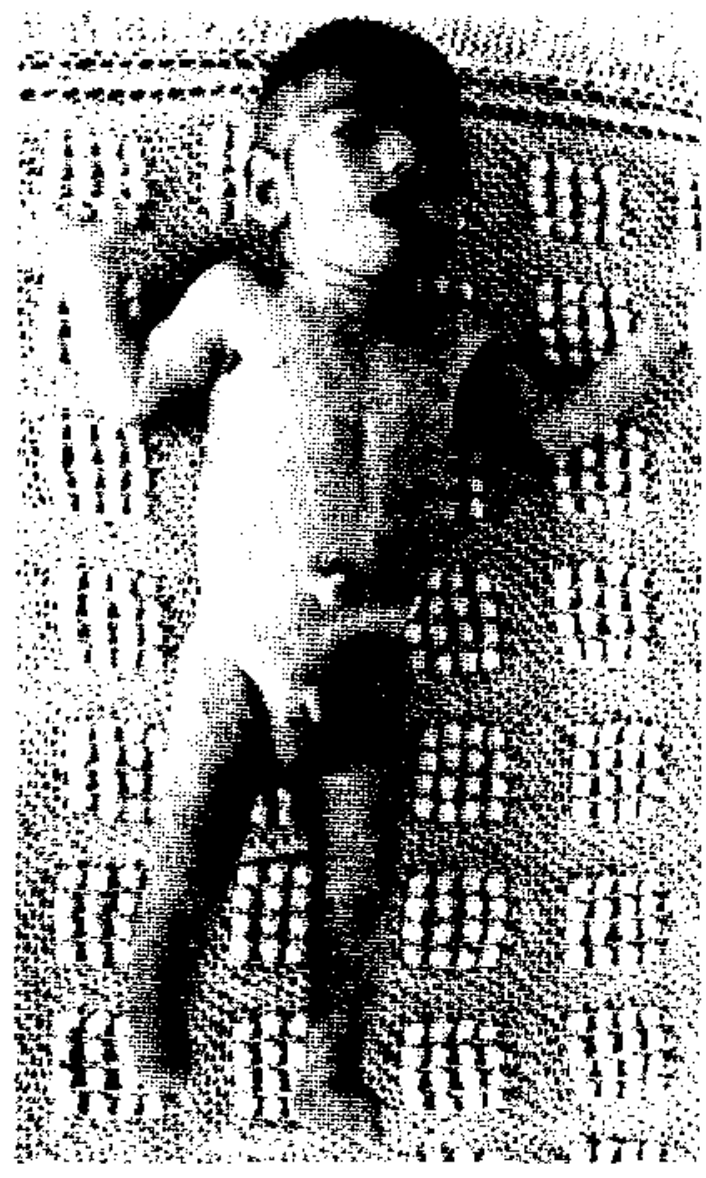

ligura 2. Cano Clinico. Insinección gieneral.

la cira. Li día 28 se rompe completamente la membrana bucofaríngea $y$ quedan en continuidad endo y ectoderma. Durante el periodo embrionatio metamórfico, que se extiende entre los días 35 y 55 , el embrión adquiere las características propias de la especie, desarrollándose su cara, cuello y miembros. La lengua se forma a partir de tres procesos pero la mayor parte de su cuerpo deriva de los procesos maxilar y mandibular. Los procesos linguales terminan de fusionarse i las ? semanas (28 días) de gestación: en la 9a semana la lengua desciende, permitiendo la disposición horizontal de los procesos palatinos y su posterior fusión.

Los esbozos de las extremidades aparecen a los 32 días, rápidamente se segmentan en 3 porciones, con una paleta distal que luego se dividirá para formar los dedos. A los 46 dias se han separado los dedos de la mano; los del pie permanecen aún unidos: El miembro superior se encuentra una semana más adelantado en sus desarrollo que el inferior.

En general, la hipoplasia y la ausencia de lengua se asocian con subdesarrollo significativo

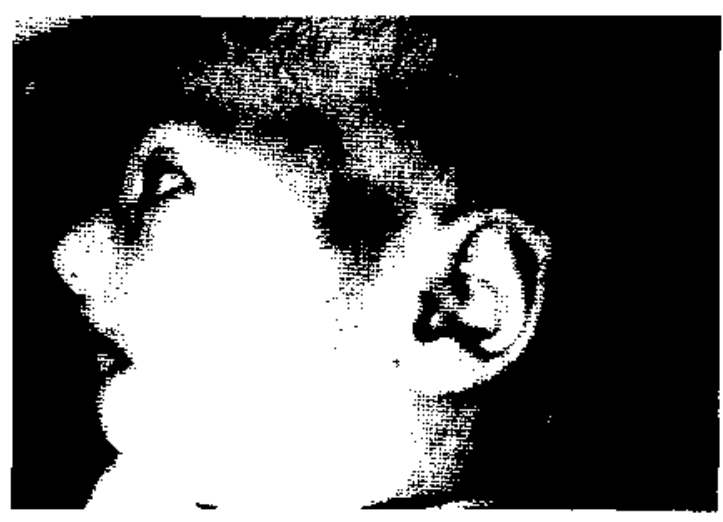

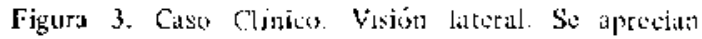
microstema za nucrestádia.

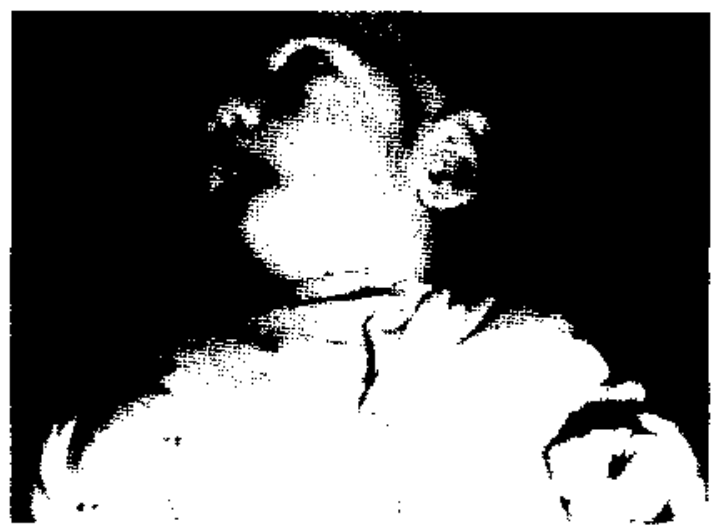

Figura 4. Castr Clinico. Detalle de ectrudactilia biluteral,

de la mandibula. En ci adulto, la membrana bucofaringea está representada por una linea en la región postetior del orofárinx entre los pilares anteriores. Posiblemente durante el desarrollo, la migración posterior esté interrumpida, resultado en la persistencia de la membrana en una posición más anterior. Este remanente pudiera causar mi. crognasia, al limitas el crecimiento de la mandibu. la; una aparente hipoglosia: por impedir la fusión ventromedial de los procesos laterales y paladar fisurado, al obstaculizar la aposición de los procesos palatinos ${ }^{14}$. Se han descrito diferentes grados de alteraciones de este tipo, desde casos que presentan una banda fibrosa fácil de seccionar hasta pacientes con anquilosis glosopalatina causada por una membrana ósea ubicada entre ambos puentes alveolares que impide la apertura de la boca ${ }^{2-5-\epsilon}$. Si se considera que esta banda corresponde a la persistencia de la membrana bucofaringea, podría estimarse que habria desaparecido más tarde que lo habitual o habría persistido hasta un periodo crítico interfiriendo el desarrollo normal.

En las extremidades el defecto puede explicar- 
se por una deficiente proliferación thesodérnica por debajo del ectoderma, la que comienza habitualmente en la 4a semana de vida embrionaria. Se trataría de un desarrollo más bien incompleto que anormal.

La etiología del Sindrome de llanhart se desconoce. No se han encontrado evidencias de agregación familiar $y$ los factores hereditarios serían poco importantes: Algunós autores han sugerido un tipo de herencia autosómica recesiva sobre la base de un caso, hijo de primos segundos, que ocurrió en una población con altas tasas de consanguinidad ${ }^{6}$. Timbién se ha planteado la posibilidad de herencia autosómica dominante con expresividad variable $y$ reducida penetrancia, por el hallazoro de alteraciones orofaciales menores en parientes del afectado ${ }^{3-15}$. Así mismo sc ha pensado que los responsables podrían ser factores ambientales intrauterinos, como hemoragias locales o bandas fibrosas mesodérmicas provenientes de las superícics cruentas después de una ruptura precoz del amnios ${ }^{16-17-19}$ : pero ambas teorias han sido abandonadas. Los estu. dios cromosónicos han sido nominas. Ambos sexos se encuentran igualmente afectados.

La prevalencia estimadia es de 1 en $500.000^{8-11}$.

En el caso que presentamos existe ol antecedente de dos familiares con defectos en el desarrollo embrionario, también liay algunos abortos espontáneos. Puđicríl plantearse alguna mayor predisposición genética a la susceptibi]idad del embrión fiente a agresiones probablemente ambientales, ya que no se ha descrito asociación entre lus sindromes de lianlhart. sindrome de Poland $y$ la presencia de labio $y$ paladar fisurados.

En cuanto a la evidencia de daño neurológico de nuestro paciente, éste probablemente tiene relación con el episodio de paro cardíaco intraoperatorio que sutrió poco después de nacer.

\section{RESUMEN}

Se presenta el caso clínico de un niño con anomalías orales y de las extrenidades concordantes con el diagnóstico de Síndrome de llanhart. En su fanilia se cncuentran otros casos de alteraciones del desarrollo.

La etiología de este síndtome se desconoce y hay extrema variabilidad en las manifestaciones clínicas.
Se discuten aspectos relativos al desarrollo embrionario que pueden ser responsables de los hallazgos en este síndrome.

\section{REFERENClA}

1. Rosenthal, R.: Aglossia congénital. A report ot the conditjon tombined with other cougenital malformjations. Am. J. Dis. Child. 44: 383, 1932.

2. Ketther: Kongenitaler Zungendefekt. Deutsthe Hedizinisthe Wocienschrit 33:532, 1907.

3. Nevin N.C., Burrows D., Allen G.. Kermalan D.C..: Aglossia adactylia syndrome. J. Med. Gener. 12:89, 1975.

4. Fulford G.E., Ardrart G.M. Kemph F.II. Aglossia congénitd. Ar il. Dis. Child. 31 : $400,1956$.

5. Pestersion (r.: Agluxsia congenjts with bony fusion of the jaws: report of one asse. Actu Chir. Seand. 122:93,1961.

6. Spivack J., Benet J.: Closiopalatine ankylosis. Plastic Reconsi. Surt. 42: 129, 1968.

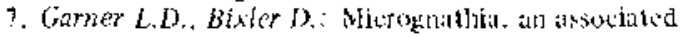
defeet of Hanltate syudrome, tipes Il and III. Orad Surgery 27: 601. 1969

8. Birth Defects Comperefian: Stsond lidition. 1979. N. York U.S.A. I'lu Nit tiolial Poundation Marcle of d)imes, Lanicl Beryumal 45 J Hanhart Syndrome.

9. Tumbilek $E$. Yalcin C., Alowe 1. : Aglossia adictylia yndrome (special emplasis on the inheritane pattern). Clin. (jenct. 11: 421, 1977.

10. Kcth E,E., Bennett C.G., Klingherg W.: Aglossiat-adactylia iyndronse. Am. J. Dis. Cluild. I] 6 : 549, 1968.

11. Harwin M. Lorinsky L.: Picture of the Month Aun. J. Jis. Child. 119: 255, 1970.

12. Hatadies oserese's de L'enfunt: Flammarion Médecine - Scientes, 1974. Pitrre Marotzaux Pistriére

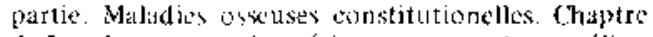
A. Les dyostoses. Agenésies irscuses, phocomélies: p. 179. Pasis, liratice

13. Birth Detects: Orjeital Article Suries, vol $\mathrm{N}^{3} 3$, March. 1969 The Clinitial Delination of Birth Defects. Part 1/1: Limb Maltormations. Congenital Skeletal Limbs Deficiencies. Newton Freire-Misia. Associated Deformitics of the Head and Hands. R.A. Pfeifter. Tle assuctation al absence deformities with orofacial malfonationes. Samia Temotauny, Victor Mc Kusjck. $\therefore$. York U.S.A.

14. Ardran G.M. Beckett J.M., Kemp F.H.: Aglossia congénita Arckl. Dis. Child. 39: 389. 1964.

15. Mendeliwn Imheritance in Mon. Catalogs of Autusomal Dominant. Autosomal Recessive and X - Linked Phenotypes. Sixth lidition, 1983, Victor Mc Kusick 10330 Aplossia adalctylia. Baltimore, Maryland L.S.A.

16. Kaplan P.M. Cumnings C., Fraser C'. A "Cummunity" of face limb malfurmation syndromes. J. Pediatr. 89: 241, 1976.

17. Poswillo $D$. . The patlrogenesis of the lirst and second branchial arth syrdrome. Oral Surgery 35: 302,1973 .

18. Nevin N.C. Dodge J.A., Kernahan D.C.: Aglossia adacty lia syndrome. Oral Surgery 29:443, 1970. 